\title{
Predictive Factors of the Presence and Number of Noncalcified Coronary Plaque in Japanese Patients with Zero Coronary Artery Calcium Score Using 64-Slice Multi-Detector Computed Tomography
}

\author{
Yoshiki Noda, Ryo Matsutera, Yoshinori Yasuoka, Kiyoshi Kume, \\ Hidenori Adachi, Susumu Hattori, Ryo Araki, Motohiro Kosugi, \\ Yasuaki Kohama, Tetsufumi Nakashima, Tatsuya Sasaki \\ Cardiovascular Division, Osaka Minami Medical Center, National Hospital Organization, Osaka, Japan \\ Email: yoshi.noda@ommc-hp.jp
}

Received June 12, 2013; revised July 11, 2013; accepted August 9, 2013

Copyright (C) 2013 Yoshiki Noda et al. This is an open access article distributed under the Creative Commons Attribution License, which permits unrestricted use, distribution, and reproduction in any medium, provided the original work is properly cited.

\begin{abstract}
Background: Factors that can predict the presence and number of noncalcified coronary plaques (NCP) in Japanese patients with zero coronary artery calcium scores (CACS) essentially remain undefined. Methods and Results: We assessed independent predictors of the presence and number of segments with NCP in 111 Japanese patients with zero CACS who underwent 64-slice multi-detector computed tomography at our hospital. Thirty five patients (32\%) had NCP, and 24 patients (22\%) had $\geq 2$ NCPs. Multiple logistic regression analysis revealed that significant predictors for the presence of NCP were age (odds ratio [OR]: 1.06, 95\% confidence interval [CI] $1.01-1.11, \mathrm{p}=0.021)$, male $(\mathrm{OR}$ : $3.61,95 \%$ CI $1.40-9.35, \mathrm{p}=0.008)$ and diabetes mellitus (OR: $3.10,95 \%$ CI $1.02-9.45, \mathrm{p}=0.046$ ), and those for the presence of $\geq 2$ NCPs were age (OR: $1.08,95 \%$ CI $1.02-1.15, \mathrm{p}=0.007)$ and a current smoking habit (OR: $5.09,95 \%$ CI $1.00-25.74, \mathrm{p}=0.049)$. Multiple linear regression analysis identified advanced age, male gender and diabetes mellitus as independent predictors of the number of NCPs. A novel score calculated from the above four predictors showed moderate accuracy for a diagnosis of NCP and $\geq 2$ NCPs, with areas under receiver operating curves of 0.738 and 0.736 , respectively. Conclusions: Male Japanese patients with zero CACS, advanced age, diabetes mellitus and a current smoking habit might have NCPs.
\end{abstract}

Keywords: Zero Coronary Artery Calcium Score; Number of Noncalcified Coronary Plaque; 64-Slice Multi-Detector Computed Tomography; Japanese Patients

\section{Introduction}

Coronary artery calcium score (CACS) is useful for the risk stratification of coronary artery disease, and zero CACS is associated with a very low likelihood of coronary artery plaque and future cardiac events $[1,2]$. The recent technical development of multi-detector computed tomography (MDCT) has rendered it feasible to calculate CACS and to detect coronary artery plaque or obstructive coronary lesions using $\mathrm{CT}$ coronary angiography (CT $\mathrm{CA})$. The presence of obstructive coronary lesions, noncalcified coronary plaque (NCP) or the number of coronary plaques detected by CTCA improves the prediction of cardiac events over and above conventional risk scores and CACS [3-6]. Moreover, NCP with both a low CT value and positive remodeling is associated with the subsequent development of acute coronary syndrome (ACS) [7].

On the other hand, several recent reports have revealed that some patients with zero CACS have NCP or significant coronary artery stenosis [8-20]. Which patients with zero CACS have NCP is important to determine so that they can derive a benefit from further examination using CTCA in addition to CAC scoring. Although some studies have identified the predictive factors of the presence of NCP in patients with zero CACS, those in Japanese patients with zero CACS have not been fully elucidated.

The aim of this study is to identify the factors that can predict the presence and number of NCPs in Japanese patients with zero CACS using 64-slice MDCT. 


\section{Methods}

\subsection{Study Population}

Between March 2009 and March 2011, 586 consecutive Japanese patients underwent 64-slice cardiac CT (both CAC scoring and CTCA) for suspected coronary artery disease (CAD) at our hospital. We excluded patients with known CAD or previous myocardial infarction, those with a history of percutaneous coronary intervention (PCI) or coronary artery bypass graft (CABG) surgery and those with unacceptable CTCA image quality. Among the remaining 353 patients, we enrolled 111 (31\%) with zero CACS. This study was approved by our institutional review board, and all patients provided written informed consent to participate.

\subsection{Cardiac CT Scanning Protocol}

The patients were evaluated by cardiac CT scanning using a 64-slice MDCT (Aquillion 64, Toshiba Medical Systems, Tochigi, Japan). Before scanning, blood pressure and heart rate were measured, sublingual nitroglycerin was administered to all of the patients, and those with an initial heart rate above 65 beats per minute were administered with beta-blockers in the absence of contraindications. Thereafter, unenhanced electrocardiographically gated CT images were acquired to calculate CACS before CTCA, which were prospectively triggered at $75 \%$ of the RR interval and obtained under the following parameters: collimation, $4 \times 3.0 \mathrm{~mm}$; gantry rotation time, $0.25 \mathrm{sec}$; tube voltage, $120 \mathrm{kV}$; and tube current, $300 \mathrm{~mA}$.

The CTCA scans were acquired as follows. Patients received an intravenous bolus of $0.9 \mathrm{~mL} / \mathrm{kg}$ (body weight) of contrast media (Iopamiron $370 \mathrm{mg} \mathrm{I} / \mathrm{mL}$; Bayer HealthCare, Osaka, Japan) at a rate of $2.5-4.5$ $\mathrm{mL} / \mathrm{sec}$, followed by $30 \mathrm{~mL}$ of saline at the same rate using a double-head injector (Dual shot ${ }^{\mathrm{TM}}$, Nemoto Kyorindou, Tokyo, Japan). A region of interest was placed within the ascending aorta, and the scan was started when the CT density reached 100 Hounsfield Units (HU) above the baseline CT density. Images were acquired under the following parameters: collimation, $64 \times 0.5$ $\mathrm{mm}$; gantry rotation time, $0.35,0.375$ or $0.4 \mathrm{sec}$; tube voltage, $120 \mathrm{kV}$; tube current, $400 \mathrm{~mA}$; beam pitch, 0.175 to 0.2 . Raw datasets of the scans were reconstructed using retrospective electrocardiogram-gated halfscan or segmental reconstruction algorithms. The optimal cardiac phase was individually determined to minimize motion artifacts.

The reconstructed data were transferred to a computer workstation (Volume Analyzer SYNAPSE VINCENT, FUJIFILM, Tokyo, Japan) for CAC scoring and NCP evaluation.

\subsection{Cardiac CT Data Analysis}

We calculated CACS using the Agatston scoring method [21] and patients with zero CACS were enrolled in this study. All CTCA images were interpreted by two experienced observers who were blinded to the clinical findings of the patients such as their characteristics, cardiovascular risk factors, clinical presentations and pretest probabilities. If the CTCA findings of the observers differed, consensus was reached and the final findings were determined. Coronary arteries were divided into 15 segments as proposed for the American Heart Association model [22], and we evaluated all segments with a diameter of $>2 \mathrm{~mm}$. Using curved multiplanar reformation (c-MPR) and cross-sectional (CS) images, we detected NCPs as structures of $>1 \mathrm{~mm}^{3}$ that could be assigned to the coronary artery walls with $\mathrm{CT}$ values below the contrast-enhanced coronary lumen but above the surrounding connective tissue, as previously described $[23,24]$. We then assessed the number of segments with NCP for each patient. Each NCP was characterized for remodeling index (RI), minimum CT value and the presence of spotty calcification (SC) and significant stenosis. We calculated RI from CS images as lesion vessel area/reference vessel area defined as the average of apparently normal proximal and distal vessel areas to the lesion. Positive remodeling (PR) was defined as RI $>1.1$. At least 5 regions of interest were placed within each NCP, and the lowest value of those was defined as the minimum $\mathrm{CT}$ value. We defined NCP with a minimum CT value of $<0 \mathrm{HU}$ as low $\mathrm{CT}$ value plaque (LP). We assessed the presence of NCPs with both PR and LP which might be associated with the subsequent development of ACS. We defined SC as being $<3 \mathrm{~mm}$ in size on c-MPR images and occupying only one side on CS images as described [7]. Stenosis was deemed significant when luminal diameter narrowing was $\geq 50 \%$.

\subsection{Cardiovascular Risk Factors and Pretest Probability}

We assessed whether patients had the following cardiovascular risk factors based on their medical records: diabetes mellitus (DM), hypertension, dyslipidemia and current smoking. DM was defined as fasting glucose $\geq$ $126 \mathrm{mg} / \mathrm{dL}$ and hemoglobin A1c $\geq 6.5 \%$ or the use of antidiabetic treatment. Hypertension was defined as systemic blood pressure $\geq 140 / 90 \mathrm{mmHg}$ or the use of antihypertensive treatment. Dyslipidemia was defined as total cholesterol $\geq 220 \mathrm{mg} / \mathrm{dL}$, low-density lipoprotein cholesterol $\geq 140 \mathrm{mg} / \mathrm{dL}$, fasting triglycerides $\geq 150$ $\mathrm{mg} / \mathrm{dL}$, high-density lipoprotein cholesterol $\leq 40 \mathrm{mg} / \mathrm{dL}$ or the use of lipid-lowering treatment. Medicines administered at the time of CT imaging were also recorded. The pretest likelihood of CAD was determined based on 
Morise scores as low $(<9)$, intermediate (9 to15) or high (> 15) [25].

\subsection{Statistical Analysis}

Continuous variables are expressed as means \pm standard deviation (SD). Categorical variables are presented as absolute numbers and relative frequencies (\%). The Chisquare test was used in univariate analyses for categorycal variables and Student's unpaired $t$ test was applied for continuous variables. Predictors of the presence of NCP, NCP with both PR and LP and $\geq 2$ NCPs were assessed using multiple logistic regression analyses, and independent predictors of the number of segments with NCP were determined using multiple linear regression analysis. The diagnostic performance of the presence of NCP and $\geq 2$ NCPs was evaluated using receiver operating characteristic (ROC) curve analyses. Cut off values were also calculated. All tests of significance were two-tailed and values of $\mathrm{P}<0.05$ were considered statistically significant. All statistical analyses were performed using Ekuseru-Toukei 2010 (Social Survey Research Information Co. Ltd., Tokyo, Japan).

\section{Results}

Table 1 shows the baseline characteristics of the enrolled patients. Over half had hypertension, dyslipidemia or chest symptoms, most (66\%) had an intermediate pretest probability of CAD, and almost all $(93 \%)$ had an intermediate or high pretest probability. Most patients were not given any medications for CAD at the time of CT imaging.

Table 2 summarizes the results of the CTCA data analyses. Of 111 patients, 35 (32\%) had NCP, 8 (7\%) had NCP with both PR and LP, and 24 (22\%) had $\geq 2$ NCPs. Twelve (11\%) patients had significant stenosis.

Independent predictors of the presence of NCP, NCP with both PR and LP and $\geq 2$ NCPs and the number of NCP were identified using multivariate analyses (Tables 3-6). Multiple logistic regression analysis revealed that significant predictors for the presence of NCP were age (odds ratio [OR]: 1.06, 95\% confidence interval [CI] 1.01 - 1.11, p = 0.021), male gender (OR: 3.61, 95\% CI 1.40 9.35, $\mathrm{p}=0.008$ ) and DM (OR: 3.10, 95\% CI $1.02-9.45$, $\mathrm{p}=0.046$ ), and those for the presence of $\geq 2$ NCPs were age (OR: $1.08,95 \%$ CI $1.02-1.15, \mathrm{p}=0.007)$ and current smoking (OR: 5.09, 95\% CI $1.00-25.74, \mathrm{p}=0.049$ ). Age, male gender and DM were independent predictors of the number of NCPs in the multiple linear regression analysis. However, no predictive factors for the presence of NCP with both PR and LP were determined by multiple logistic regression analysis. Other factors, such as body mass index (BMI), hypertension, dyslipidemia, presence of chest symptom and Morise pretest score, were
Table 1. Baseline characteristics of the patients $(n=111)$.

\begin{tabular}{|c|c|}
\hline Variable & Value \\
\hline Age, years & $61.5 \pm 10.8$ \\
\hline Male, $\mathrm{n}$ & $43(39 \%)$ \\
\hline Body mass index, $\mathrm{kg} / \mathrm{m}^{2}$ & $23.5 \pm 3.3$ \\
\hline Diabetes mellitus, $\mathrm{n}$ & $19(17 \%)$ \\
\hline Hypertension, $\mathrm{n}$ & $62(56 \%)$ \\
\hline Dyslipidemia, $\mathrm{n}$ & $55(50 \%)$ \\
\hline Current smoking, $\mathrm{n}$ & $12(11 \%)$ \\
\hline Chest symptom, $n$ & $72(65 \%)$ \\
\hline \multicolumn{2}{|l|}{ Medication, $\mathrm{n}$} \\
\hline Aspirin & $12(11 \%)$ \\
\hline ACE-I or ARB & $21(19 \%)$ \\
\hline Statins & $25(23 \%)$ \\
\hline Beta-blockers & $7(6.3 \%)$ \\
\hline Morise pretest score & $13.7 \pm 3.4$ \\
\hline Low risk, $\mathrm{n}$ & $8(7 \%)$ \\
\hline Intermediate risk, $\mathrm{n}$ & $73(66 \%)$ \\
\hline High risk, $\mathrm{n}$ & $30(27 \%)$ \\
\hline
\end{tabular}

Values are expressed as means $\pm \mathrm{SD}$. ACE-I, angiotensin converting enzyme inhibitors; ARB, angiotensin II receptor blockers.

Table 2. Numbers of patients with significant stenoses or NCPs $(\mathbf{n}=111)$.

\begin{tabular}{cc}
\hline Variable & Value \\
\hline Significant stenosis $(\geq 50 \%)$ & $12(11 \%)$ \\
NCP & $35(32 \%)$ \\
With PR & $15(14 \%)$ \\
With LP & $14(13 \%)$ \\
With both PR and LP & $8(7.2 \%)$ \\
With SC & $1(0.9 \%)$ \\
$\geq 2$ NCPs & $24(22 \%)$
\end{tabular}

LP, low CT value plaque; NCP, non-calcified coronary plaque; PR, positive remodeling; SC, spotty calcification.

not associated with the presence or number of NCPs.

We developed a novel score for predicting NCP or $\geq 2$ NCPs in patients with zero CACS derived from the results of these analyses. Among the four predictors, age was considered the most significant based on a standard- 
Table 3. Factors predicting the presence of NCP.

\begin{tabular}{|c|c|c|c|c|}
\hline & \multicolumn{2}{|c|}{ Univariable } & \multicolumn{2}{|c|}{ Multivariable } \\
\hline & OR $(95 \% \mathrm{CI})$ & $\mathrm{p}$ value & OR $(95 \% \mathrm{CI})$ & $\mathrm{p}$ value \\
\hline Age & $1.03(0.99-1.08)$ & 0.107 & $1.06(1.01-1.11)$ & 0.021 \\
\hline Male & $3.07(1.34-7.04)$ & 0.008 & $3.61(1.40-9.35)$ & 0.008 \\
\hline Diabetes mellitus & $3.90(1.40-10.83)$ & 0.009 & $3.10(1.02-9.45)$ & 0.046 \\
\hline Hypertension & $2.18(0.94-5.07)$ & 0.070 & $1.39(0.55-3.52)$ & 0.491 \\
\hline Current smoking & $2.41(0.72-8.11)$ & 0.154 & $2.28(0.51-10.14)$ & 0.278 \\
\hline
\end{tabular}

CI, confidence interval; OR, odds ratio.

Table 4. Factors predicting the presence of NCP with both PR and LP.

\begin{tabular}{ccccc}
\hline & Univariable & & \multicolumn{2}{c}{ Multivariable } \\
\hline Age & OR $(95 \%$ CI $)$ & p value & OR $(95 \%$ CI $)$ & p value \\
Male & $0.99(0.93-1.06)$ & 0.787 & $1.02(0.94-1.11)$ & 0.628 \\
Diabetes mellitus & $2.85(0.64-12.60)$ & 0.167 & $2.39(0.41-13.96)$ & 0.332 \\
Dyslipidemia & $3.26(0.71-15.03)$ & 0.129 & $1.91(0.34-10.76)$ & 0.463 \\
Current smoking & $3.31(0.64-17.15)$ & 0.155 & $3.27(0.57-18.64)$ & 0.182 \\
\hline
\end{tabular}

CI, confidence interval; OR, odds ratio.

Table 5. Factors predicting the presence of $\geq 2$ NCPs.

\begin{tabular}{ccccc}
\hline & Univariable & \multicolumn{2}{c}{ Multivariable } \\
\hline Age & OR $(95 \%$ CI $)$ & p value & OR $(95 \%$ CI $)$ & p value \\
Male & $1.06(1.01-1.11)$ & 0.026 & $1.08(1.02-1.15)$ & 0.007 \\
Diabetes mellitus & $1.81(0.73-4.50)$ & 0.204 & $1.83(0.63-5.30)$ & 0.266 \\
Current smoking & $2.57(0.88-7.51)$ & 0.084 & $2.50(0.68-9.22)$ & 0.170 \\
Statins & $3.01(0.86-10.52)$ & 0.085 & $5.09(1.00-25.74)$ & 0.049 \\
\hline
\end{tabular}

CI, confidence interval; OR, odds ratio.

Table 6. Factors predicting the number of NCP.

\begin{tabular}{cccc}
\hline & Regression Coefficient & SE & p value \\
\hline Age & 0.025 & 0.010 & 0.016 \\
Male & 0.767 & 0.233 & 0.001 \\
Diabetes mellitus & 0.965 & 0.294 & 0.001 \\
Statins & -0.466 & 0.263 & 0.079 \\
\hline
\end{tabular}

SE, standard error.

ized partial regression coefficient. Therefore, we assigned higher scores for age and defined the novel score as age (years) $/ 10+$ male gender $(1 / 0)+\mathrm{DM}(1 / 0)+$ current smoking $(1 / 0)$ and then evaluated the diagnostic performance of the score using ROC curve analyses (Figure 1). The novel score was more accurate for diagnosing the presence of NCP and $\geq 2$ NCPs with areas under receiver operating curves (AUCs) of 0.738 and 0.736 , respectively, than Morise scores, with AUCs of 0.666 and 0.652 , respectively. The optimal cut-off points of the novel score to predict the presence of NCP and $\geq 2$ NCPs were 7.0 with a sensitivity of $71 \%$ and a specificity of $71 \%$ and 6.7 with a sensitivity of $83 \%$ and a specificity of $55 \%$, respectively.

Figure 2 shows a 67-year-old patient who had 3 NCPs 
including one with both PR and LP. His novel score of 9.7 was calculated as age (67)/10 + male gender (1) + DM (1) + current smoking (1). He underwent PCI for the obstructive NCP lesion with both PR and LP in left anterior descending artery (Figures 2 (b) and (c)), and then received intensive medical therapy.

\section{Discussion}

The main findings of the present study were (1) quite a few Japanese patients with zero CACS had NCP, among whom some had NCP with both PR and LP, which might be associated with ACS, and others had $\geq 2$ NCPs. (2) Advanced age, male gender, DM and a current smoking habit were independent predictors of the presence of NCPs and the number of NCPs in Japanese patients with zero CACS.

Although zero CACS has been associated with a very low likelihood having of coronary artery plaque and significant $\mathrm{CAD}$, as well as a minimal risk of future cardiovascular events $[1,2]$, recent reports have indicated that some patients with zero CACS do indeed have NCP or significant coronary artery stenosis. The reported prevalence of NCP and obstructive coronary lesions in patients with zero CACS is $7 \% \sim 51 \%$ and $<1 \% \sim 24 \%$, respectively [8-20]. The variation is thought to be due to differences in patients' characteristics, such as clinical pre-

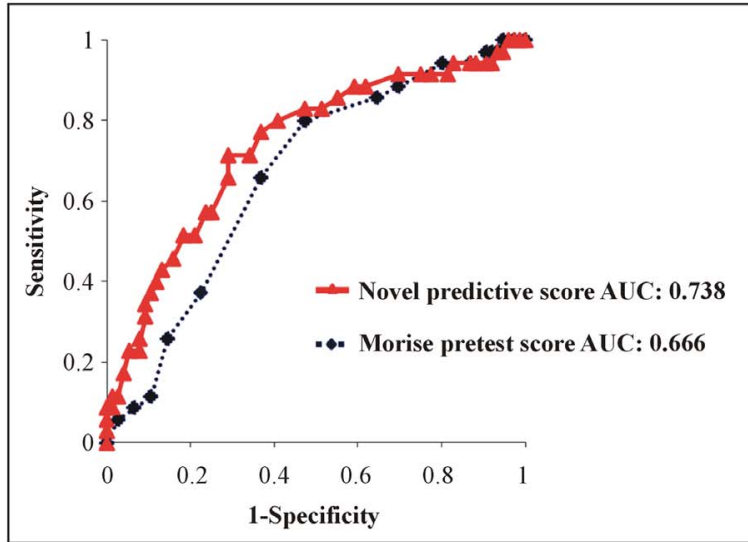

(a)

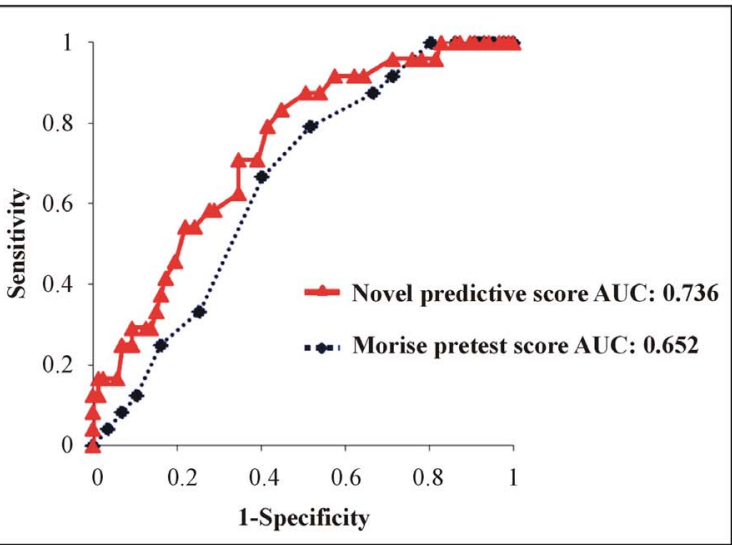

(b)

Figure 1. ROC curve analyses of the novel and Morise pretest scores for predicting the presence of NCP (a) and $\geq 2$ NCPs (b). AUC, area under receiver operating curve; NCP, non-calcified coronary plaque; ROC, receiver operating characteristic.

(a)

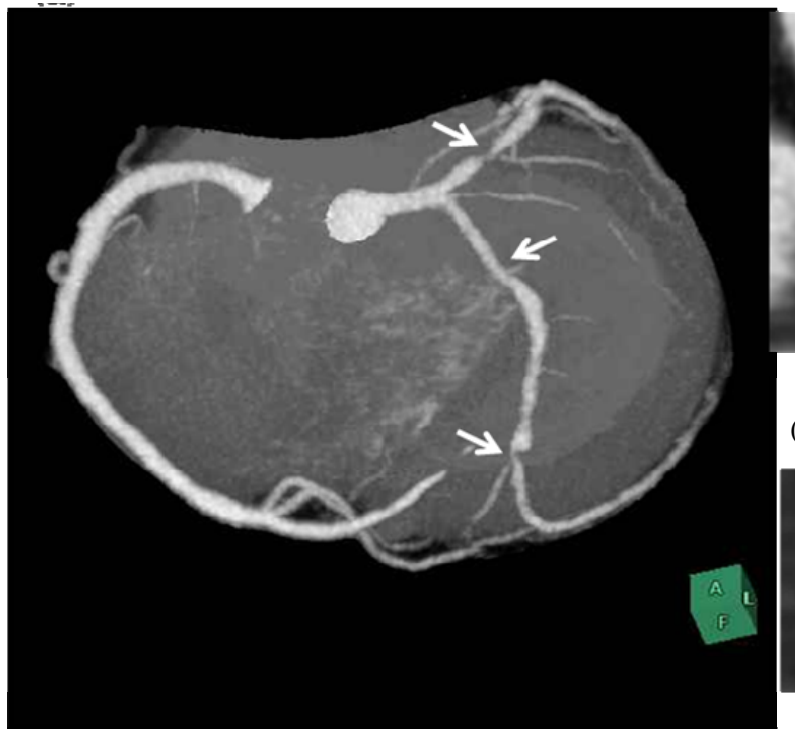

(b)

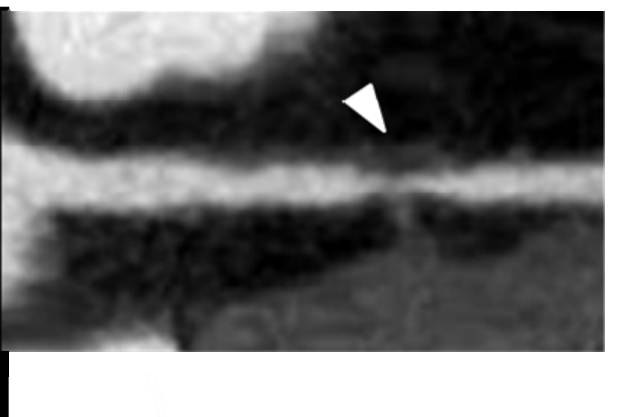

(c)

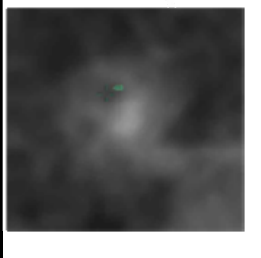

Figure 2. Representative 67-year-old male patient with zero CACS and 3 NCPs. Angiographic view (a) shows 3NCPs (arrows). One in left anterior descending artery has positive remodeling and minimum CT value of $<0$ HU on c-MPR (b, arrowhead) and CS (c) image. His novel predictive score was 9.7. CACS, coronary artery calcium score; c-MPR, curved multiplanar reformation; CS, cross-sectional; NCP, non-calcified coronary plaque. 
sentation, race, gender and risk factors $[9,19]$, and generally, reviewing the above recent reports, NCP or obstructive coronary lesions appear more frequent in study populations at higher risk for CAD. On the other hand, there are not many studies with Asian, especially Japanese patients, which reveal that the prevalence of NCP and obstructive coronary lesions in patients with zero CACS is $12 \%$ and $2.4 \% \sim 3.1 \%$, respectively $[11,17,19]$. The present study found that $11 \%$ of the patients without CAC had significant stenosis, $32 \%$ of them had NCP, and $7 \%$ of them had NCP with both PR and LP. Their prevalence was much higher than those previously reported about Japanese patients. This might be because many of our patients had chest symptoms $(65 \%)$ and an intermediate or high pretest probability (93\%), indicating that we evaluated more patients at higher risk for cardiovascular disease compared with previous studies.

Predictive factors for the presence of NCP in patients with zero CACS have also been described in several articles. Ergün et al. reported that age and DM are associated with positive CTCA findings [14]. Uretsky et al. reported that age ( $>60$ years), male gender and a current smoking history are associated with a greater tendency to have coronary plaque [15]. Chen et al. reported that age $(>55$ years) and BMI ( $>27)$ are independent factors associated with the presence of plaques [18]. In the study with Japanese patients, Ueda et al. identified hypercholesterolemia and DM as independent predictors of NCP or mixed coronary plaque [11]. Morita et al. also identified age and male gender as significant predictors of 2 feature-positive plaque having positive remodeling and low attenuation, which is associated with the subsequent development of ACS [19]. We similarly found that advanced age, male gender, DM and current smoking were independent predictors of the presence of NCPs in patients with zero CACS. However, our multivariate analysis could not identify predictors of NCP with both PR and LP. This might be because only a few patients had NCP with both PR and LP. Further investigation of a larger study cohort is needed to demonstrate predictive factors for NCP with both PR and LP.

Several studies have indicated a higher prevalence of significant $\mathrm{CAD}$ without $\mathrm{CAC}$ for patients below 50 years of age [16,26-28]. Moreover, zero CACS might reliably exclude obstructive CAD among older patients $[16,26,29]$. In contrast, none of 12 patients who had significant stenosis without CAC were below 50 years old in the present study and advanced age was one of the independent predictors of the presence of NCPs. Multivariate analysis in some studies including one with Japanese patients has associated advanced age with the presence of plaques in patients with zero CACS, as described above. The present findings agree with these. The cause of this inconsistency has not been clearly elucidated, but it might be due to the selection criteria and the small proportions of patients with zero CACS in the present and in these studies.

A recent study using the Framingham risk score found that intermediate to high-risk scores comprised an independent risk factor for NCP in patients with zero CACS [10]. In addition, many investigators have found that the prevalence of obstructive CAD in patients with zero CACS and a high suspicion of CAD is not negligible $[9,12,20]$. However, the Morise pretest scores in the present multivariate analyses did not comprise an independent predictor of the presence of NCPs, and did not have high diagnostic sensitivity for the presence of NCP and $\geq 2$ NCPs on ROC curve analyses (Figure 1). Although one of the reasons for this discrepancy could be the small SD in the Morise scores of our population (SD = 3.4) (Table 1), conventional pretest scores might be limited for predicting the presence of NCP because they were developed using conventional coronary angiography as a reference, but this cannot detect nonluminal narrowing NCP. New pretest scores are needed that can predict the presence of NCP. We therefore propose using a novel score for predicting NCPs with moderate diagnostic performance in patients with zero CACS. However, another prospective study is required to validate this novel score.

Another point of the present study is the number of NCP detected by CTCA. Hadamitzky et al. reported that the number of coronary segments with plaque detected by CTCA had incremental predictive value for future cardiac events over the Morise score and CACS [5]. Lin et al. examined mortality risk in relation to the extent and composition of nonobstructive plaques using CTCA in a prospective two-center study [30]. They found that individuals with $>5$ segments containing nonobstructive plaque had an increased intermediate term mortality hazard, compared with those with $<5$ segments. These findings suggest that the number of coronary segments containing plaque should be an important predictor of future cardiovascular events. However, to our knowledge, the number of coronary segments with NCP in patients with zero CACS has not been described. Here, we identified age, male gender and DM as predictors of the number of NCP in Japanese patients with zero CACS. Although in the present study, these predictors were the same as those of the presence of NCP, another predictor, a current smoking habit was one of the independent predictors of $\geq$ 2 NCPs. A recent study has demonstrated that smokers with zero CACS have higher relative mortality than nonsmokers with zero CACS [31]. Our results suggest that smoking might lead to coronary plaque progression, resulting in an increased risk of mortality in the patients with zero CACS.

Although the present results suggest recommending 
CTCA for some patients even if their CACS is zero, radiation exposure due to CTCA remains a concern. The mean radiation exposure of CTCA estimated as the doselength product (DLP) was $1354 \pm 145 \mathrm{mGy} \cdot \mathrm{cm}$. This figure was relatively higher than in previous CTCA studies [32]. The radiation dose should be minimized without degradation of image quality using various techniques, such as the appropriate reduction of tube voltage or tube current, prospective electrocardiogram-gated scanning and iterative reconstruction techniques [33,34]. Moreover, the development of CT hardware, such as wider coverage scanners, faster gantry rotation speeds and higher pitch scanning should enable further significant reductions in radiation doses [34].

Our study has several limitations. First, all of our patients were selected from a single center, they were of the same ethnic background, or Japanese patients, and the study cohort was quite small. Thus, our results cannot be directly extrapolated to the general population. Further studies including large cohorts from several centers and multiethnic populations are needed to generalize the present results. Second, we did not perform invasive coronary angiography including intravascular ultrasound, which should be the gold standard with which to confirm the presence of coronary plaque. Current 64-slice MDCT has limited spatial resolution for assessing coronary plaque. Further improvement of the spatial resolution of MDCT is desirable. Third, we did not investigate the outcomes of the present study population. Few reports have described the relationship between the presence of NCP and future cardiac events in patients with zero CACS. Outcome analysis is required to define the diagnostic significance of detecting NCP in patients with zero CACS.

\section{Conclusion}

Even if CACS was zero, the presence of NCP could not be excluded, especially in male Japanese patients with advanced age, diabetes mellitus and a current smoking habit. Among the patients with zero CACS and NCP, some had NCP with both PR and LP that might be associated with ACS, and those with $\geq 2$ NCPs. Our results suggest that these patients should undergo CTCA in addition to CAC scoring from the viewpoint of coronary risk stratification. In the future, in order to demonstrate the importance of this study, we need to analyze cardiovascular outcomes in Japanese patients with zero CACS and NCP.

\section{REFERENCES}

[1] P. Greenland, R. O. Bonow, B. H. Brundage, M. J. Budoff, M. J. Eisenberg, S. M. Grundy, et al., "ACCF/ AHA 2007 Clinical Expert Consensus Document on
Coronary Artery Calcium Scoring by Computed Tomography in Global Cardiovascular Risk Assessment and in Evaluation of Patients With Chest Pain: A Report of the American College of Cardiology Foundation Clinical Expert Consensus Task Force (ACCF/AHA Writing Committee to Update the 2000 Expert Consensus Document on Electron Beam Computed Tomography) Developed in Collaboration With the Society of Atherosclerosis Imaging and Prevention and the Society of Cardiovascular Computed Tomography," Journal of the American College of Cardiology, Vol. 49, No. 3, 2007, pp. 378-402. doi:10.1016/j.jacc.2006.10.001

[2] A. Sarwar, L. J. Shaw, M. D. Shapiro, R. Blankstein, U. Hoffmann, R. C. Cury, et al., "Diagnostic and Prognostic Value of Absence of Coronary Artery Calcification," JACC: Cardiovascular Imaging, Vol. 2, No. 6, 2009, pp. 675-688. doi:10.1016/j.jcmg.2008.12.031

[3] G. Pundziute, J. D. Schuijf, J. W. Jukema, E. Boersma, A. de Roos, E. E. van der Wall, et al., "Prognostic Value of Multislice Computed Tomography Coronary Angiography in Patients With Known or Suspected Coronary Artery Disease," Journal of the American College of Cardiology, Vol. 49, No. 1, 2007, pp. 62-70. doi:10.1016/j.jacc.2006.07.070

[4] V. Russo, A. Zavalloni, M. L. Bacchi Reggiani, K. Buttazzi, V. Gostoli, S. Bartolini, et al., "Incremental Prognostic Value of Coronary CT Angiography in Patients With Suspected Coronary Artery Disease," Circulation: Cardiovascular Imaging, Vol. 3, 2010, pp. 351-359. doi:10.1161/CIRCIMAGING.109.880625

[5] M. Hadamitzky, R. Distler, T. Meyer, F. Hein, A. Kastrati, S. Martinoff, et al., "Prognostic Value of Coronary Computed Tomographic Angiography in Comparison With Calcium Scoring and Clinical Risk Scores," Circulation: Cardiovascular Imaging, Vol. 4, 2011, pp. 16-23. doi:10.1161/CIRCIMAGING.110.955351

[6] C. F. de Azevedo, M. S. Hadlich, S. G. Bezerra, J. L. Petriz, R. R. Alves, O. de Souza, et al., "Prognostic Value of CT Angiography in Patients With Inconclusive Functional Stress Tests," JACC: Cardiovascular Imaging, Vol. 4, No. 7, 2011, pp. 740-751. doi:10.1016/i.jemg.2011.02.017

[7] S. Motoyama, M. Sarai, H. Harigaya, H. Anno, K. Inoue, T. Hara, et al., "Computed Tomographic Angiography Characteristics of Atherosclerotic Plaques Subsequently Resulting in Acute Coronary Syndrome," Journal of the American College of Cardiology, Vol. 54, No. 1, 2009, pp. 49-57. doi:10.1016/j.jacc.2009.02.068

[8] J. L. Kelly, D. Thickman, S. D. Abramson, P. R. Chen, S. F. Smazal, M. J. Fleishman, et al., "Coronary CT Angiography Findings in Patients Without Coronary Calcification," American Journal of Roentgenology, Vol. 191, No. 1, 2008, pp. 50-55. doi:10.2214/AJR.07.2954

[9] I. Gottlieb, J. M. Miller, A. Arbab-Zadeh, M. Dewey, M. E. Clouse, L. Sara, et al., "The Absence of Coronary Calcification Does Not Exclude Obstructive Coronary Artery Disease or the Need for Revascularization in Patients Referred for Conventional Coronary Angiography," Journal of the American College of Cardiology, Vol. 55, No. 7, 2010, pp. 627-634. doi:10.1016/j.jacc.2009.07.072 
[10] M. Alqarqaz, M. Zaidan and M. H. Al-Mallah, "Prevalence and Predictors of Atherosclerosis in Symptomatic Patients With Zero Calcium Score", Academic Radiology, Vol. 18, No. 11, 2011, pp. 1437-1441. doi:10.1016/j.acra.2011.07.012

[11] H. Ueda, K. Harimoto, S. Tomoyama, H. Tamaru, M. Miyawaki, N. Mitsusada, et al., "Association between Cardiovascular Risk Factors and the Presence of Coronary Plaque in a Zero or Low Coronary Artery Calcium Score," International Journal of Cardiology, Vol. 147, No. 3, 2011, pp. 475-477. doi:10.1016/j.ijcard.2011.01.026

[12] T. C. Villines, E. A. Hulten, L. J. Shaw, M. Goyal, A. Dunning, S. Achenbach, et al., "Prevalence and Severity of Coronary Artery Disease and Adverse Events Among Symptomatic Patients With Coronary Artery Calcification Scores of Zero Undergoing Coronary Computed Tomography Angiography: Results From the CONFIRM (Coronary CT Angiography Evaluation for Clinical Outcomes: An International Multicenter) Registry," Journal of the American College of Cardiology, Vol. 58, No. 24, 2011, pp. 2533-2540. doi:10.1016/j.jacc.2011.10.851

[13] D. H. Yoo, E. J. Chun, S. I. Choi, J. A. Kim, K. N. Jin, T. J. Yeon, et al., "Significance of Noncalcified Coronary Plaque in Asymptomatic Subjects With Low Coronary Artery Calcium Score: Assessment With Coronary Computed Tomography Angiography," International Journal of Cardiovascular Imaging, Vol. 27, No. 1, 2011, pp. 27-35. doi:10.1007/s10554-011-9968-1

[14] E. Ergün, P. Koşar, C. Oztürk, E. Başbay, F. Koç and U. Koşar, "Prevalence and Extent of Coronary Artery Disease Determined by 64-Slice CTA in Patients With Zero Coronary Calcium Score," International Journal of Cardiovascular Imaging, Vol. 27, No. 3, 2011, pp. 451-458. doi:10.1007/s10554-010-9681-5

[15] S. Uretsky, A. Rozanski, P. Singh, A. Supariwala, P. Atluri, S. Bangalore, et al., "The Presence, Characterization and Prognosis of Coronary Plaques Among Patients With Zero Coronary Calcium Scores," International Journal of Cardiovascular Imaging, Vol. 27, No. 6, 2011, pp. 805812. doi:10.1007/s10554-010-9730-0

[16] Y. E. Yoon, S. A. Chang, S. I. Choi, E. J. Chun, Y. S. Cho, T. J. Youn, et al., "The Absence of Coronary Artery Calcification Does Not Rule Out the Presence of Significant Coronary Artery Disease in Asian Patients With Acute Chest Pain," International Journal of Cardiovascular Imaging, Vol. 28, No. 2, 2012, pp. 389-398. doi:10.1007/s10554-011-9819-0

[17] H. Ueda, K. Harimoto, S. Tomoyama, H. Tamaru, M. Miyawaki, N. Mitsusada, et al., "Relation of Cardiovascular Risk Factors and Angina Status to Obstructive Coronary Artery Disease According to Categorical Coronary Artery Calcium Score," Heart Vessels, Vol. 27, No. 2, 2012, pp. 128-134. doi:10.1007/s00380-011-0128-2

[18] C. K. Chen, Y. S. Kuo, C. A. Liu, M. H. Sheu, H. T. Chang, C. W. Chen, et al., "Frequency and Risk Factors Associated With Atherosclerotic Plaques in Patients With a Zero Coronary Artery Calcium Score," Journal of the Chinese Medical Association, Vol. 75, No. 1, 2012, pp. 10-15. doi:10.1016/j.jcma.2011.11.001
[19] H. Morita, S. Fujimoto, T. Kondo, T. Arai, T. Sekine, H. Matsutani, et al., "Prevalence of Computed Tomographic Angiography-Verified High-Risk Plaques and Significant Luminal Stenosis in Patients With Zero Coronary Calcium Score," International Journal of Cardiology, Vol. 158, No. 2, 2012, pp. 272-278. doi:10.1016/j.ijcard.2011.02.052

[20] Y. J. Kim, J. Hur, H. J. Lee, H. J. Chang, J. E. Nam, Y. J. Hong, et al., "Meaning of Zero Coronary Calcium Score in Symptomatic Patients Referred for Coronary Computed Tomographic Angiography," European Heart Journal: Cardiovascular Imaging, Vol. 13, No. 9, 2012, pp. 776-785. doi:10.1093/ehjci/jes060

[21] A. S. Agatston, W. R. Janowitz, F. J. Hildner, N. R. Zusmer, M. Viamonte Jr. and R. Detrano, "Quantification of Coronary Artery Calcium Using Ultrafast Computed Tomography," Journal of the American College of Cardiology, Vol. 15, No. 4, 1990, pp. 827-832. doi:10.1016/0735-1097(90)90282-T

[22] W. G. Austen, J. E. Edwards, R. L. Frye, G. G. Gensini, V. L. Gott, L. S. Griffith, et al., "A Reporting System on Patients Evaluated for Coronary Artery Disease. Report of the Ad Hoc Committee for Grading of Coronary Artery Disease, Council on Cardiovascular Surgery, American Heart Association," Circulation, Vol. 51, 1975, pp. 5-40. doi:10.1161/01.CIR.51.4.5

[23] A. W. Leber, A. Knez, A. Becker, C. Becker, F. von Ziegler, K. Nikolaou, et al., "Accuracy of Multidetector Spiral Computed Tomography in Identifying and Differentiating the Composition of Coronary Atherosclerotic Plaques: A Comparative Study With Intracoronary Ultrasound," Journal of the American College of Cardiology, Vol. 43, No. 7, 2004, pp. 1241-1247.

doi:10.1016/j.jacc.2003.10.059

[24] S. Achenbach, F. Moselewski, D. Ropers, M. Ferencik, U. Hoffmann, B. MacNeill, et al., "Detection of Calcified and Noncalcified Coronary Atherosclerotic Plaque by Contrast-Enhanced, Submillimeter Multidetector Spiral Computed Tomography: A Segment-Based Comparison with Intravascular Ultrasound," Circulation, Vol. 109, 2004, pp. 14-17. doi:10.1161/01.CIR.0000111517.69230.0F

[25] A. P. Morise, W. J. Haddad and D. Beckner, "Development and Validation of a Clinical Score to Estimate the Probability of Coronary Artery Disease in Men and Women Presenting With Suspected Coronary Disease," American Journal of Medicine, Vol. 102, No. 4, 1997, pp. 350-356. doi:10.1016/S0002-9343(97)00086-7

[26] K. Akram, R. E. O'Donnell, S. King, H. R. Superko, A. Agatston and S. Voros, "Influence of Symptomatic Status on the Prevalence of Obstructive Coronary Artery Disease in Patients With Zero Calcium Score," Atherosclerosis, Vol. 203, No. 2, 2009, pp. 533-537. doi:10.1016/j.atherosclerosis.2008.07.008

[27] M. Marwan, D. Ropers, T. Pflederer, W. G. Daniel and S. Achenbach, "Clinical Characteristics of Patients with Obstructive Coronary Lesions in the Absence of Coronary Calcification: An Evaluation by Coronary CT Angiography," Heart, Vol. 95, 2009, pp. 1056-1060. doi: $10.1136 / \mathrm{hrt} .2008 .153353$

[28] A. Knez, A. Becker, A. Leber, C. White, C. R. Becker, M. 
F. Reiser, et al., "Relation of Coronary Calcium Scores by Electron Beam Tomography to Obstructive Disease in 2,115 Symptomatic Patients," American Journal of Cardiology, Vol. 93, No. 9, 2004, pp. 1150-1152. doi:10.1016/j.amjcard.2004.01.044

[29] A. Becker, A. Leber, C. W. White, C. Becker, M. F. Reiser and A. Knez, "Multislice Computed Tomography for Determination of Coronary Artery Disease in a Symptomatic Patient Population," International Journal of Cardiovascular Imaging, Vol. 23, No. 3, 2007, pp. 361-367. doi:10.1007/s10554-006-9189-1

[30] F. Y. Lin, L. J. Shaw, A. M. Dunning, T. M. Labounty, J. H. Choi, J. W. Weinsaft, et al., "Mortality Risk in Symptomatic Patients With Nonobstructive Coronary Artery Disease: A Prospective 2-Center Study of 2,583 Patients Undergoing 64-Detector Row Coronary Computed Tomographic Angiography," Journal of the American College of Cardiology, Vol. 58, No. 5, 2011, pp. 510-519. doi:10.1016/j.jacc.2010.11.078

[31] J. W. McEvoy, M. J. Blaha, J. J. Rivera, M. J. Budoff, A. N. Khan, L. J. Shaw, et al., "Mortality Rates in Smokers and Nonsmokers in the Presence or Absence of Coronary
Artery Calcification," JACC: Cardiovascular Imaging, Vol. 5, No. 10, 2012, pp. 1037-1045. doi:10.1016/j.jemg.2012.02.017

[32] J. Hausleiter, T. Meyer, F. Hermann, M. Hadamitzky, M. Krebs, T. C. Gerber, et al., "Estimated Radiation Dose Associated With Cardiac CT Angiography," JAMA, Vol. 301, No. 5, 2009, pp. 500-507. doi:10.1001/jama.2009.54

[33] J. Leipsic, T. M. Labounty, B. Heilbron, J. K. Min, G. B. Mancini, F. Y. Lin, et al., "Estimated Radiation Dose Reduction Using Adaptive Statistical Iterative Reconstruction in Coronary CT Angiography: The ERASIR Study," American Journal of Roentgenology, Vol. 195, No. 3, 2010, pp. 655-660. doi:10.2214/AJR.10.4288

[34] S. S. Halliburton, S. Abbara, M. Y. Chen, R. Gentry, M. Mahesh, G. L. Raff, et al., "SCCT Guidelines on Radiation Dose and Dose-Optimization Strategies in Cardiovascular CT," Journal of Cardiovascular Computed Tomography, Vol. 5, No. 4, 2011, pp. 198-224. doi:10.1016/j.jcct.2011.06.001 